\title{
Parametric Study and Simulation of Horizontal Split Casing Pump by using Computational Fluid Dynamics
}

\author{
Ramesh. S. Gavade, Suresh M. Sawant
}

\begin{abstract}
The pump requirement is required to meet the specified performance parameters means the required pressure (head) and flow, using less power. For water pump applications, periodic maintenance is required to check worn parts such as wear ring, shaft sleeves. In such a case the direct separation case pumps are reliable. If a given work point can meet the range of pumps of the available separate cases, it is necessary to develop a new pump and the upgraded pump should be more efficient with the considered power problems. With the help of computational fluid dynamics, the complex internal flow of the pump in the case of equilibrium separation can be accurately predicted, thus facilitating the construction of the pump. Simulation provides the most complete performance testing and important diagnostic details that help the engineer quickly and increase composition. As a result, the company is able to quickly create new designs such as a fully patented water pump with a patent. Pressure suction can be problematic during operation and operation of centrifugal pumps. Such pressure stimulants are traditionally investigated but quantitative analysis techniques allow these effects to be tested. Numerical analysis is done with a multi-reference framework to predict the flow field within the entire pipeline and impeller.
\end{abstract}

Key Words- Centrifugal pump, CFD, BEP, CAE, RANS model

\section{INTRODUCTION}

The centrifugal pump is a hydrodynamic pump, in which the rotating air continuously transfers the work of the machine from the drive to the liquid. The kinetic energy is converted into potential pressure potential. Computational Fluid Dynamics (CFD) is one of the tools of Computer Aided Engineering (CAE). CFD has recently emerged as another way to investigate the complex state of water flow in pumps. It soon becomes an important tool for analysis and design in water engineering. Input to CAE software is meshed geometry of the model and this geometric modeling is done using Computer Aided Design (CAD) software. The centrifugal pump, the most widely used type of pump to date, is an important member of the turbo machine family for moving drinks from the lowest to the highest levels. Like the standard type of centrifugal pump,

Revised Manuscript Received on December 25, 2020.

* Correspondence Author

Ramesh S. Gavade*, Research Scholar, Department of Mechanical Engineering, RIT Rajaramnagar, Shivaji University, Kolhapur (M.H) India, Email: rameshaditiom21@gmail.com

Suresh M. Sawant, Department of Mechanical Engineering, RIT Rajaramnagar, Shivaji University, Kolhapur (M.H) India, Email: suresh.sawant@ritindia.edu

(c) The Authors. Published by Blue Eyes Intelligence Engineering and Sciences Publication (BEIESP). This is an open access article under the CC BY-NC-ND license (http://creativecommons.org/licenses/by-nc$\mathrm{nd} / 4.0 /$ ) double-centrifugal suction pump with high transition and high weight are broadly utilized in the business. Nonetheless, customary strategies for pump development and effectiveness particularly embrace an experimentation technique by delivering and testing model pump, which are costly and tedious. Albeit high productivity appeared to be the main capacity of pump that could create critical financial advantages, other execution pointers were likewise significant. Nourbakhsh et.al. [1] presented techniques that give the cycle to choosing the fitting PAT for a particular little force station. Contingent upon the test information, one technique utilizing a deliberate point can be set as follows. The evaluated point is the working climate of the machine, contingent upon the site conditions and the recurrence isn't actually predictable with the plan point or BEP of the machine. Chua et.al. [2] examined the stream designs inside the divergent blood stream hallways. Four distinct kinds of edge calculation have been tried including straight spiral sharp edge and back scope plans. Both of these plans can arrive at a head of around $100 \mathrm{~mm} \mathrm{Hg}$ in a plan climate that is compelling in improving pump execution with ease. Zhang et.al. [3] another plan strategy for making a twofold divergent pump was proposed, and it was demonstrated that RBFs joined with the pump execution model models were created, and progressed Pareto arrangements were gotten utilizing MOEA/D, which uncovered clashes between center capacities, productivity, and NPSHr. Spenc et.al [4] A two fold digit mathematical model, a volute radial pump was utilized to perform parametric investigations including four huge mathematical boundaries. The boundaries incorporate a water cutting hole, a vane game plan, a snubber hole and a sidewall expulsion, orchestrated in three distinct designs utilized for every boundary. A sum of 33 impermanent exchanges have been handled, indicating 45,000 h of persistent examination time and including in excess of $550 \mathrm{~GB}$ of investigation and results information. Anagnostopoulos et.al. [5] show the mathematical technique for figuring the stream field.The outward pump transmission pump and the pump activity bend are created, controlled, and assessed against the test and factual information, with empowering results. The proposed stream estimation calculation is ideal for hydrodynamic developments in water driven pump and turbines, because of the quick and programmed age of the Cartesian matrix and expanded precision in speaking to abnormal boundaries.

Published By:

Blue Eyes Intelligence Engineering and Sciences Publication

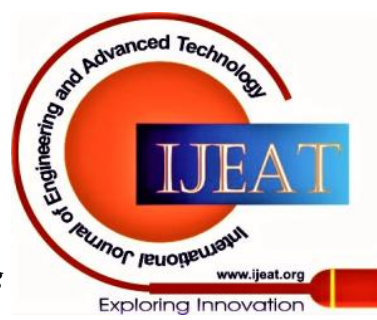

(C) Copyright: All rights reserved. 
The current examination is that the strategy gives the capacity to localize the optimal range of the free design variables at low computer cost.

Reeves et.al [6] Imitation provides the most complete performance testing and critical diagnostic details that help the engineer quickly and increase composition. As a result, the company is able to quickly create new designs such as a fully patented water pump with a patent. The new design uses a mixing flow area associated with the material and diffuser resulting in a more efficient construction that improves pump performance and can eliminate mechanical signals currently accounting for approximately 95 percent of all pump warranty claims. Hornsby et.al [7] A remarkable breakthrough in recent years is that of unconventional technology that allows for true modeling of geometric engineering instead of restricting the use of simpler methods in systematic ways. Coupling this with the expansion of portable models, which allow for multiphase flow to be studied, and the investigation of cavitation-like conditions in new and existing pump designs becomes a viable proposal. It available to meet revised air emissions objectives. The result of this change is that engine manufacturers rely heavily on suppliers to provide engineering and production assistance to the peripheral components and the EMP has accelerated their product development efforts to address these needs. The company's research and development space provides a focused and creative environment away from the daily worries of production. At KSB Aktiengesellschaft in Frankenthal, Germany, CFD was utilized to test the exhibition of the new diffuser and packaging plan for their heater siphon. Measurements demonstrated a decrease in all out weight misfortune by practically half of new development contrasted with the past one, which prompted critical upgrades in siphon effectiveness. In many creating lands, more modest force stations are required. For instance, an attainability study indicated that in excess of 200 force stations could be introduced in Iran [1]. Utilizing a siphon as a turbine (PAT) is another appealing and significant technique. Siphons are moderately straightforward machines that are extraordinarily planned and promptly accessible in many creating lands. It should be noticed that the radial siphon may have some improvement over other siphon plans, for example, the hub (or inclining) siphon. Despite the fact that the size of the pivotal siphon can be kept more modest than the radiating siphon explored here, to accomplish a similar stream rate at $5 \mathrm{l} / \mathrm{min}$ the speed of the hub siphon should increment. A multi-stage configuration may should be considered to accomplish a worthy topic. This can prompt high shear pressure (or at the degree of hemolysis) of the blood gracefully to the siphon. On the whole stream way the game plan of the vane is a conspicuous boundary despite the fact that this standard abatements as the stream rate diminishes, particularly in regions close to cutting water. The cutting water hole shows a little commitment, yet this increments as the stream rate diminishes with the territories around the cutting water. The snubber hole and sidewall expulsion ordinarily have almost no effect, in spite of the fact that this can be generally recognizable at low stream levels.

Presently a day the vast majority of the necessities for the utilization of high-volume and yield water administrations are between $1020 \mathrm{~m} 3 / \mathrm{hr}$ to $2520 \mathrm{~m} 3 / \mathrm{hr}$. The current

Retrieval Number: 100.1/ijeat.B20001210220

DOI:10.35940/ijeat.B2000.1210220

Journal Website: www.ijeat.org prerequisite for head focuses $(\mathrm{H})$ is $216 \mathrm{~m}$ and yield $(\mathrm{Q})$ is $2150 \mathrm{~m} 3 / \mathrm{hr}$. The scope of the current case siphon model is up to $160 \mathrm{~m}$ head of $2150 \mathrm{~m} 3 / \mathrm{hr}$. It is in this manner important to redesign the siphon for another very good quality arrangement of head-to-run pulls. Simultaneously with power issues it is expected that the updated siphon should utilize less force for proficiency.

Different sorts of siphons, for example, multistage siphon, Vertical Turbine (VT) siphon, blended stream (MF) siphon can be provided with the necessary current capacity of high head and release. Flat Split case siphons are entirely appropriate for water flexibly plot anticipates for the accompanying reasons:

It is as basic in plan as the correlation of a multistage siphon with a Vertical turbine siphon.

Components that pivot gradually and are anything but difficult to gather.

Low manufacturing costs.

Simple to keep up.

For as far back as 25 years, CFD has been broadly utilized in an assortment of designing applications. At first, the utilization of these strategies was conventional just in the field of aviation and atomic. From that point, utilization spread over an assortment of items, states of being, and creation measures. On account of siphon area applications, CFD apparatuses are significant when considering siphon pressure measure. With impeller entrance, CFD can help improve the progression of infiltration stream by planning/precisely surveying the stream pace of the siphon [7 - 8]. Test and scholastic examination requires extensive time. Also, in testing the genuine visual model of the model is needed for costly creation. As CFD investigation should be possible in kind as well. CFD apparatuses abstain from demonstrating and ordinary testing. The better and quicker plan of the siphon and its examination lead to more limited development cycles.

\section{FLOW SIMULATION OF PUMP}

In order to obtain better design in CFD, following procedure is applied so that fluid flow can easily be modeled in the centrifugal pump impeller.

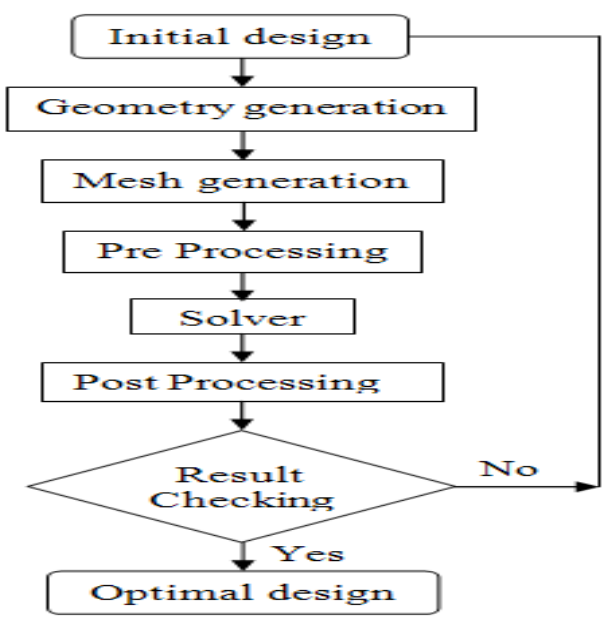

Fig.1. Flow chart of CFD procedure

It is now feasible to use CFD

Published By:

Blue Eyes Intelligence Engineering and Sciences Publication

(C) Copyright: All rights reserved.

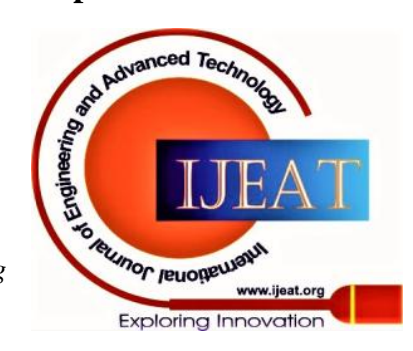


codes for a realistic prediction of the complex three dimensional (3D) turbulent flow in the entire pump. The 3D views of hydraulic flow passage of the suction volute, impeller and delivery volute as shown in Fig. 2.

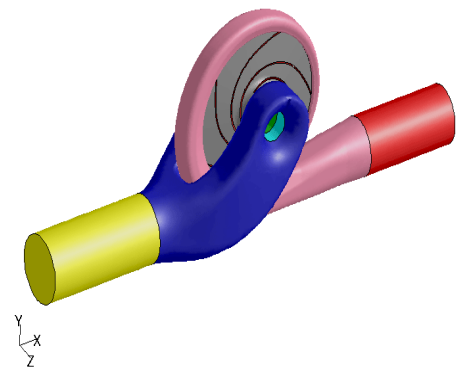

Fig.2. Flow domains of pump for CFD

3D flow simulation is performed for the passage of hydraulic flow. Numerous numerical calculations were performed to predict the flow pattern within all pliers and boats, in which the flow impeller field is solved by a circular frame and a single letter [9]. The two reference frames are connected in such a way that each has a fixed position in all calculations. The appropriate change occurs in a smooth visual interface without a visual limit. An impeller is used with a specific speed of 51.8 metric unit. The main pump parameters are presented in Table.1.

Table.1. Geometrical Parameters of the pump

\begin{tabular}{|lll|}
\hline \multicolumn{2}{c|}{ Impeller } \\
\hline Parameter & Value & Description \\
D2 & $825 \mathrm{~mm}$ & Impeller outside diameter \\
$\beta 1$ & $38^{\circ}$ & Blade inlet angle \\
$\beta 2$ & $71^{\circ}$ & Blade outlet angle \\
b2 & $27 \mathrm{~mm}$ & Impeller Outlet Width \\
D1 & $310 \mathrm{~mm}$ & Impeller Eye Diameter \\
Su & $6 \mathrm{~mm}$ & Blade Thickness \\
z & 5 & Number of Blades \\
\hline \multicolumn{3}{c|}{ Volute } \\
\hline Parameter & Value & Description \\
b3 & $54 \mathrm{~mm}$ & Volute inlet width \\
D3 & $865 \mathrm{~mm}$ & Cut water diameter \\
$\varnothing$ outlet & $310 \mathrm{~mm}$ & Volute outlet diameter \\
\hline
\end{tabular}

It has been found that good volute formation can improve pump performance and operating scope [1-4]. Voltage studies are performed on existing geometries and can be divided into two groups: volute flow analysis and flow communication between volute and impeller. There are five parameters of geometrical key in volute formation: circular variations of the cross-sectional area; cross-sectional formation; the position spread across the cross section; the position of the volute inlet; and language geometry [4 - 5]. The need for construction to create a circulating pressure distribution uniform.

\section{Computational Domain, Grid Generation and boundary Conditions:}

The first task to accomplish on numerical flow simulation is the definition of the geometry, followed by the grid generation. A structured grid is created using the HyperMesh software as shown in Fig.3. The grid of total flow domain passages has 55860 nodes. The blades, hub and shroud surfaces and casings surfaces are modeled as solid walls. All periodic grid surfaces are defined as periodic boundary conditions. Finally mesh file is imported in fluent software.

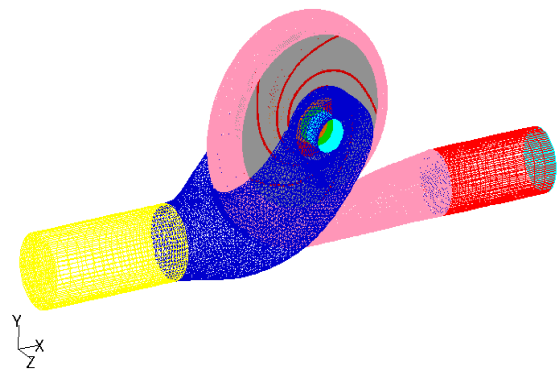

Fig.3. Meshed model for CFD analysis

The general parameters and boundary conditions used for the 3D flow simulation of the pump are summarized in Table.2.

Table.2. Simulation Parameters used in Fluent

\begin{tabular}{|ll|}
\hline \multicolumn{1}{|c|}{ Parameters } & \multicolumn{1}{c|}{ Description } \\
\hline $\begin{array}{l}\text { Flow simulation } \\
\text { domain }\end{array}$ & $\begin{array}{l}\text { Extended Inlet and outlet, } \\
\text { Suction volute, Delivery } \\
\text { volute and impeller }\end{array}$ \\
Grid & Structured \\
Fluid & Water at room temperature \\
Inlet & Mass flow= 2150 \\
Outlet & $\mathrm{m}^{3} / \mathrm{hr}=597.22 \mathrm{~kg} / \mathrm{s}$ \\
Turbulence Model & Pressure outlet \\
Discretization & Standard k-epsilon model \\
Maximum residual & Second order upwind \\
conversions criteria & $10^{-4}$ (RSM) \\
\hline
\end{tabular}

The imitation base in the channel and source segments was wide enough to take into account the redundancy of the bay and the elliptic effect of the stream. The CFD code stream arrangement utilizes the insecure progression of aggravation of the ceaseless number. Three variables impact picking a model of confusion: (1) the idea of the issue, (2) the nature of the outcomes visited, and (3) the processing power. Contingent upon the seriousness of the stream, this choice is consistently significant when utilizing CFD codes. Notwithstanding, the customary Reynolds Averaged Navier Stoke (RANS) model, the standard k-c thick model is utilized [10]. The dividers are displayed utilizing the log-law divider work. Transport measurements are arranged utilizing the last volume strategy. In the current examination, the standards of shift in weather conditions in accumulated insights are reenacted utilizing the second most precise framework for trading the top characterization framework with the remedy of body publicizing. At the section of the PC space, the weight stream proportion in the full record outline, the extent of the aggravation, the scale and the reference pressure in one network territory are indicated.

Published By:

Blue Eyes Intelligence Engineering DOI:10.35940/ijeat.B2000.1210220 and Sciences Publication (C) Copyright: All rights reserved.

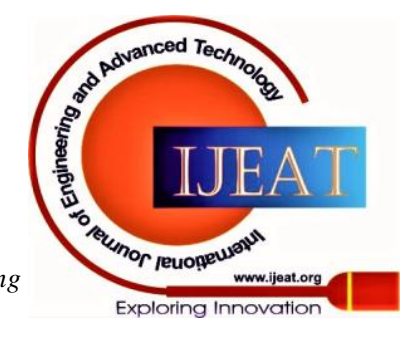


The speed vector is in the hub heading. Cutting edges, harp and cover region and zones of housings are made as strong dividers. Each time framework territory is characterized as intermittent limit conditions. Fluid liquid factors, for example, discharge pressure and velocity are discharged from the flight outlet of the exhaust pipe section [11 - 12].

\section{RESULTS}

The whole three-dimensional flow field in centrifugal impeller, volute and circling casings, is obtained by numerical analysis. Conclusive circuits of total pressure and velocity distribution between the length of the pumping area and the volute casings in the design area are shown in Fig. 4. According to Fig. 5 Complete pressure distribution shows a slight difference in the impeller area. CFD simulation provides a visual image of the internal flow of a machine that allows for more complex analysis and understanding. While the effect of arrogant language is appealing, the distribution of the same pressure is the same as around the river. The flow pattern in the casings also affects the performance of the pump.

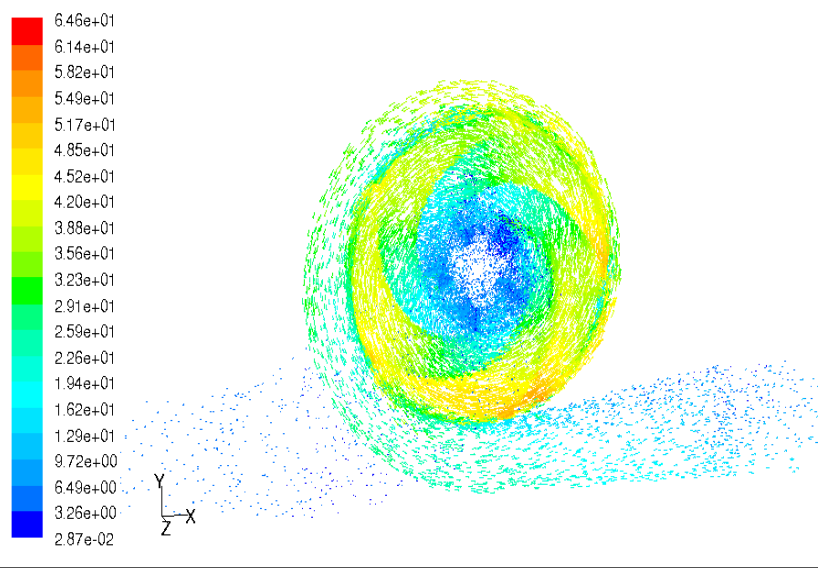

Fig.4. Velocity Vectors Colored by Velocity Magnitude $(\mathbf{m} / \mathbf{s})$

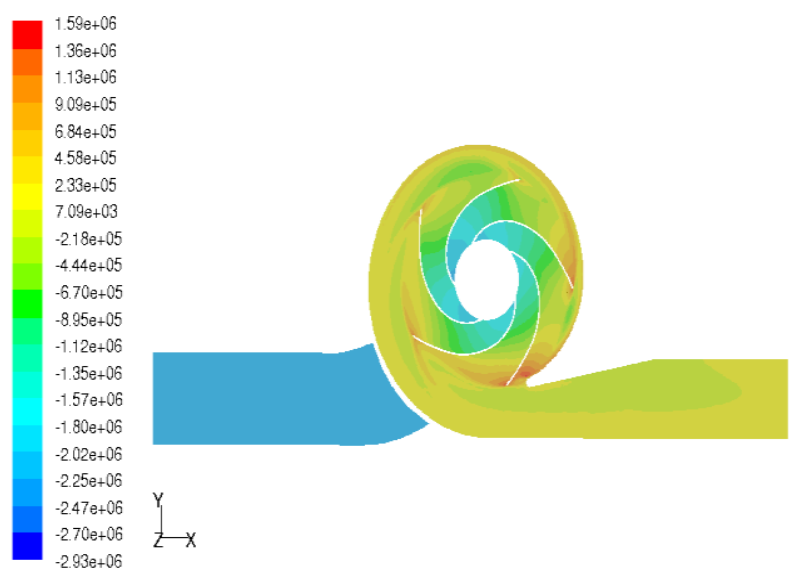

Fig.5. Contours of Total Pressure field on middle plane

The torque volute language is found corresponding to the combination of the smallest area, the largest area, and the exit lump. At this point, the volute flow meets the dividing plane, below where the flow exits the pump, above where the flow enters the volute. The leading edge of the tongue is the same as the air, the leading edge has a lower closure but is sensitive to the part of the attack; while a well-led edge is highly restrictive but does not care about events. This difference manifests itself as a design tradeoff. A sharp volute tongue provides perfect performance, while a volute with a rounded tongue has better composing performance.

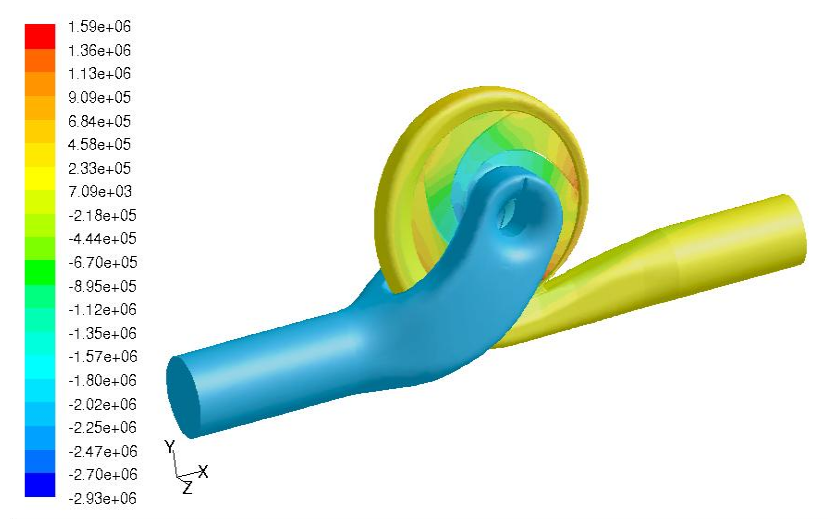

Fig.6. Contours of Total Pressure

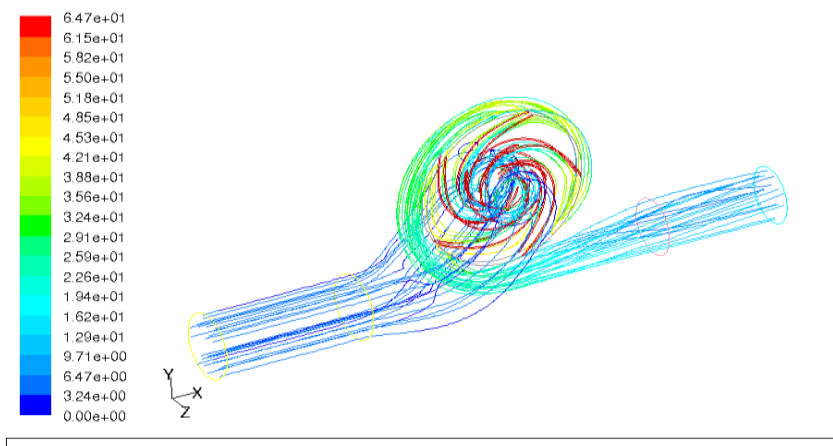

Fig.7. Path lines colored by velocity magnitude

Comparison between CFD analysis and Experimental testing:

Comparison between the CFD analysis and experimental results for the hydraulic performance of the Pump is as shown in Table.3 and Fig.8.

Table.3. Comparison between CFD analysis and Experimental testing

\begin{tabular}{|l|l|l|l|}
\hline Sr. & Parameters & CFD Analysis & $\begin{array}{l}\text { Experimental } \\
\text { Results }\end{array}$ \\
\hline 1 & Discharge & $2150 \mathrm{~m}^{3} / \mathrm{hr}$ & $2150 \mathrm{~m}^{3} / \mathrm{hr}$ \\
\hline 2 & Total head & $220 \mathrm{~m}$ & $219.7 \mathrm{~m}$ \\
\hline 3 & Pump input & $1570.88 \mathrm{~kW}$ & $1579.33 \mathrm{~kW}$ \\
\hline 4 & Efficiency & $82 \%$ & $81.45 \%$ \\
\hline
\end{tabular}

\begin{tabular}{|c|c|}
\hline KIRLOSKAR BROTHERS LIMITED & CURVE NO. 17312313 \\
\hline FPERFORMANCE CURVE OF PUMP SCT $300 / 82$ & SUC. $350 \mathrm{~mm}$ DEL. $300 \mathrm{~mm}$ \\
\hline THIS CURVE RELATES TO LIQUID OF SP. GR. $=1.0$ & \\
\hline
\end{tabular}

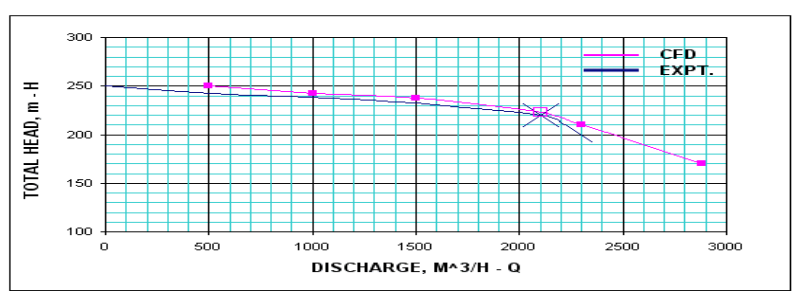

Fig.8. H-Q characteristics using CFD and Experimental testing

Published By:

Blue Eyes Intelligence Engineering and Sciences Publication

(C) Copyright: All rights reserved.

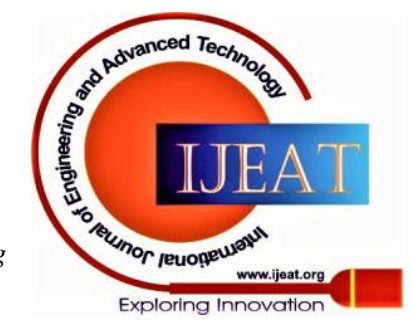


Performance found satisfactory as per the Acceptance standard IS 9137.

\section{CONCLUSION}

The flow analysis described above has been developed / adopted by Indian Pipeline Manufacturers. This has helped them to improve the reliability, safety, and efficiency of the pumping system and to develop the design feature that makes their pumps more expensive from a life cycle cost perspective. The adjacent nearby pump has been modified by increasing the width to $820 \mathrm{~mm}$ from $770 \mathrm{~mm}$ to suit the high efficiency, required head and outlet. Considering the economic motivation of the degree of efficiency and effectiveness is obtained by a better understanding of the influence of language. A very efficient pump and a stable operating circuit is designed. CFD analysis of the pump with the modified diameter of the pump was performed to check the efficiency and efficiency of the pump. The efficiency of the pump from the results of the CFD comes in at $82 \%$ and with the actual performance of the performance test it comes in at $81.45 \%$, which ensures that the CFD analysis is clearly verified.The traditional design is based on dual analysis, and is emphasized on the collection (Impeller) and less on the performance of diffusion (Volute). However, with the use of advanced modeling tools, it is possible to design a volute using threedimensional analysis. The design and analysis of the volumes presented is part of a new product being developed at Kirloskar Brothers Limited.

\section{REFERENCES}

1. Ahmad Nourbakhsh "Experimental study of characteristic curves of centrifugal pumps in different specific speeds". Journal of Experimental Thermal and Fluid Science 32, 2008, pp. 800-807.

2. W.K. Chan, L.P. Chua-"The flow patterns within the impeller passages of a centrifugal pump model". Journal of Medical Engineering and Physics 22, 2000, pp. 381-393.

3. S. Ahmad Nourbakhsh, "Experimental and Finite Element Method (FEM) failure analysis and optimization of centrifugal pump volute casing”. Journal of Engineering Failure Analysis 16, 2009.

4. Dr. Ing Friedrich, "Economic development of efficient centrifugal pump impellers by numerical methods". World Pumps Magazine May 2001, pp. 48-53.

5. R. Spence, "CFD for parametric study of geometrical variations on the pressure pulsations and performance characteristics of a centrifugal pump". Journal of Computers and Fluids 38, 2009, pp. 1243-1257.

6. John S. Anagnostopoulos, "Numerical method for flow analysis and blade design in centrifugal pump impeller". Journal of Computers and Fluids 38, 2009, pp. 284-289.

7. Chris Reeves, "Computer simulation helps design more efficient water pumps”. World Pumps Magazine, June 2004, pp. 32-34.

8. Craig Hornsby, "CFD-driving pump design forward". World Pumps Magazine, August 2002, pp. 18-22.

9. Val S. Lobanoff and Robert R. Ross, "Centrifugal Pumps design and application", Jaico publishing house, 1995.

10. A. J. Stepanoff, "Centrifugal and Axial flow pumps - Theory, Design and Application", John Wiley and Sons, 1953.

11. Stephen Lazarkiewicz and Adam T. Troskolanski, "Impeller pumps", Pergramon Publication, 1965.

12. Shigley J.E. and Mischeke C.R., "Mechanical Engineering Design", Tata McGraw-Hill Book Company, 2003.

13. John D. Anderson, "Computational Fluid Dynamics-The Basics with Applications” Taylor and Francis Publications, 1993.

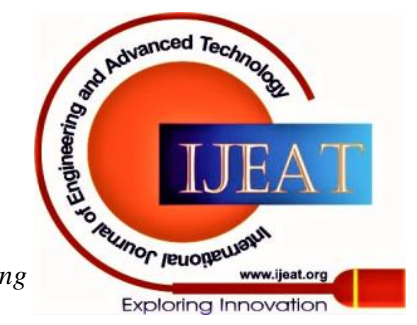

